\title{
Tomographic PIV measurements and RANS simulations of secondary flows inside a horizontally positioned helically coiled tube
}

\author{
Péter Kováts $^{1} \cdot$ Fabio J. W. A. Martins ${ }^{1,2} \cdot$ Michael Mansour $^{1,3} \cdot$ Dominique Thévenin $^{1} \cdot$ Katharina Zähringer $^{1} \mathbb{D}$
}

Received: 17 October 2019 / Revised: 25 March 2020 / Accepted: 2 April 2020 / Published online: 16 April 2020

(C) The Author(s) 2020

\begin{abstract}
Helically coiled tubes are widely used in industry to enhance heat and mass transfer in the laminar flow regime, due to their secondary flow pattern. In this study, tomographic particle image velocimetry (tomo-PIV) is used in a horizontally coiled helical tube to systematically acquire 3C-3D velocity fields for Reynolds numbers ranging from 20 to 1400 and Dean numbers from 8 to 567. The velocity field evaluations are performed using two different approaches: time-averaged velocity field calculation from instantaneous velocity fields and velocity field determination by cross-correlation from an ensemble of instantaneous reconstructed volumes. Equivalent velocity field accuracy is achieved in both velocity approaches when the flow can be considered stationary. Moreover, numerical simulations were carried out in the same geometry at the same flow conditions and were validated against the experimental 3C-3D data sets. The simulation results show good agreement with the measured velocities, offering the possibility of parametric studies and design optimization. To the authors' best knowledge, this is the first systematic experimental investigation of a helical coil flow by means of 3C-3D velocity measurements, which results can now be used for validation of numerical models in computational fluid dynamics.
\end{abstract}

\section{Graphic abstract}

Measured time-averaged velocity visualized by vector-magnitude colour at horizontal and vertical slices (left) and Dean vortices detected by 3D Q-criterion (right) from the time-averaged measurements (purple isosurfaces) and from the simulation (red isosurfaces) inside the helically-coiled tube at $\mathrm{Re}=220$ and $\mathrm{De}=89$.

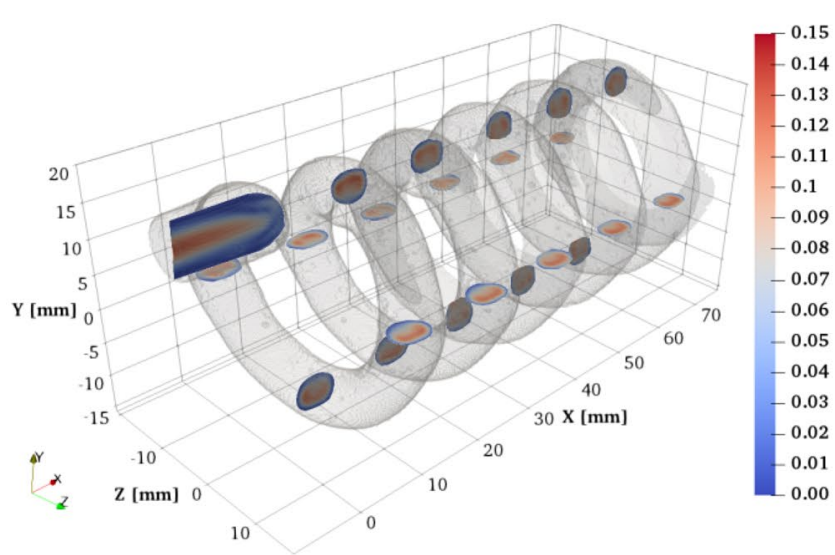

Electronic supplementary material The online version of this article (https://doi.org/10.1007/s00348-020-02950-6) contains supplementary material, which is available to authorized users.

Katharina Zähringer

katharina.zaehringer@ovgu.de

Extended author information available on the last page of the article

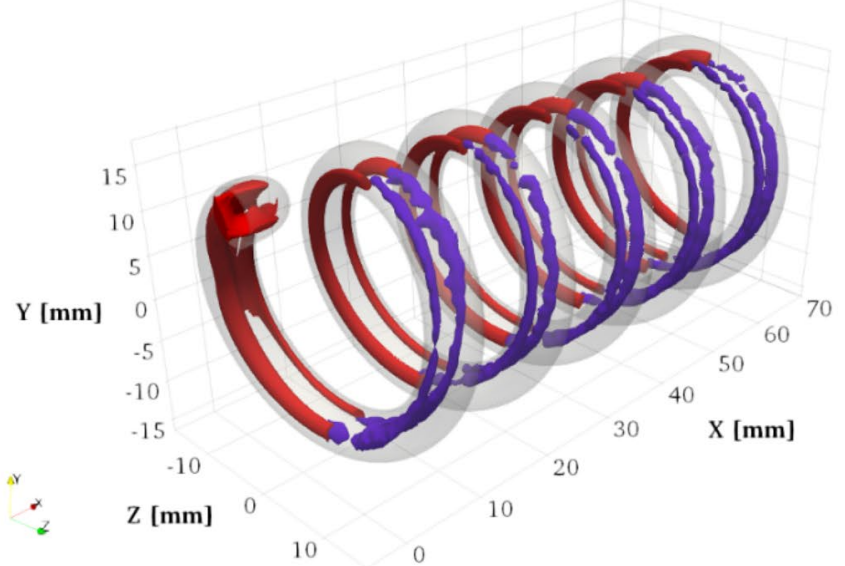

\begin{tabular}{ll}
\multicolumn{2}{l}{ List of symbols } \\
$b(\mathrm{~mm})$ & Coil pitch \\
$d(\mathrm{~mm})$ & Inner tube diameter \\
$D(\mathrm{~mm})$ & Coil diameter \\
$n(-)$ & Refractive index \\
$Q(\mathrm{ml} / \mathrm{min})$ & Volume flow rate
\end{tabular}




$\begin{array}{ll}\nu(\mathrm{m} / \mathrm{s}) & \text { Fluid bulk velocity } \\ \lambda(-) & \text { Torsion-to-curvature ratio } \\ \mu(\operatorname{Pa~s}) & \text { Dynamic viscosity } \\ \nu\left(\mathrm{m}^{2} / \mathrm{s}\right) & \text { Kinematic viscosity } \\ \rho\left(\mathrm{kg} / \mathrm{m}^{3}\right) & \text { Density } \\ \operatorname{De}(-) & \text { Dean number } \\ \operatorname{Re}(-) & \text { Reynolds number }\end{array}$

\section{Introduction}

Coiled tubes are widely used in both laboratory and industrial applications. This tube configuration is particularly interesting for reactor applications in the laminar flow regime, because complex secondary flow structures can be achieved without internal installations and with low energy costs (Kurt et al. 2015; Liang and Zhang 2014; Liou 1992; Mori and Nakayama 1967; Saxena and Nigam 1983). These helical structures enhance radial mixing and improve mass transfer as well as heat transfer compared to those of a straight pipe, e.g. conventional tube reactors (Acharya et al. 1992; Kováts et al. 2018; Moulin et al. 1996; Tohidi et al. 2015; Vashisth et al. 2008). The importance of understanding these secondary flow structures is extended also to the human body, where curved and coiled tubes can be found in the form of blood vessels (Bulusu and Plesniak 2018; Ku 1997) and bronchi (Soni et al. 2009), for example.

The flow structures developed though curved tubes started to be experimentally investigated in the early twentieth century by Eustice (Eustice 1997). After this study, Dean carried out extensive research on curved pipes (Dean 1927, 1928). He concluded, after solving the Navier-Stokes equations for laminar flows through round tube cross sections, that, due to the curvature of the tube, the flow is influenced by the centrifugal force, which leads to strong secondary flow structures in the form of twin counter rotating vortices (currently known as Dean vortices). This secondary flow is characterized by the Dean number and by the torsionto-curvature ratio. The Dean number expresses the relative magnitudes of inertial and centrifugal forces to viscous forces, and it is defined as $\mathrm{De}=\mathrm{Re} \cdot \sqrt{d /\left(D(1+\lambda)^{2}\right)}$ (Mishra and Gupta 1979), where $d$ is the inner tube diameter and $D$ is the coil diameter. The torsion-to-curvature ratio characterizes the helical geometry, and it is defined as $\lambda=b /(\pi D)$, where $\mathrm{b}$ is the coil pitch. Taylor (Taylor 1997) observed that the critical Reynolds number for turbulent flow is much higher in a curved geometry than in a straight pipe, due to the Dean vortices.

Besides these early investigations, the flow field through curved tubes has been scarcely studied afterwards by experiments. Laminar and turbulent flow velocities were measured by means of Pitot tube (Adler 1934) and by hot-wire anemometer in curved pipes (Yasuo and Wataru 1965). Axial dispersion, so important in some chemical processes, was examined extensively in a variety of helical coils with colorimetry (Koutsky and Adler 1964). Flow velocities through curved pipes (Agrawal et al. 1978; Enayet et al. 1982; Sudo et al. 1998, 2000) and through helically coiled geometries (Liou 1992) were measured using laser Doppler anemometry (LDA), a one-dimensional non-intrusive optical technique. Also the transition from laminar to turbulent flow in helically coiled pipes was studied by measuring the friction factor (Cioncolini and Santini 2006). One supposes that transition is pushed to higher Reynolds numbers, but a final validation of this statement is missing.

In the recent years, the development of particle image velocimetry (PIV) allowed to optically measure velocity fields inside complex geometries in two dimensions. Planar PIV (two components, 2C) and its stereoscopic version (three components, 3C) were used to analyse turbulent flows in $90^{\circ}$ pipe bends and to determine the flow pattern and velocity profiles (Kalpakli and Örlü 2013; Kalpakli Vester et al. 2016; Sakakibara and Machida 2012). The transient flow through a helically coiled pipe was additionally characterized by the aid of PIV (Brito et al. 2016). More recently, magnetic resonance velocimetry (MRV) technique was employed to measure the velocity in a helically coiled membrane contactor (Mejia Mendez et al. 2017) and in a $180^{\circ}$ curved artery model (Bulusu and Plesniak 2018).

This literature review shows that systematic measurements of velocity fields in helically coiled structures are, even in the laminar regime, rare; three-dimensional and three-component (3C-3D) measurements are nearly inexistent, although they are very important for the validation of the numerous existing numerical studies. From the abovementioned non-intrusive methods, just MRV is capable to obtain the three velocity components within a three-dimensional volume simultaneously. Nevertheless, MRV needs a great number of data acquisitions of the same flow over a long time period in order to achieve good spatial resolution, which makes the technique restricted to steady or periodical flows (Fukushima 1999).

Contrarily, tomographic PIV (Elsinga et al. 2006; Raffel et al. 2018) technique does not have this limitation. Nevertheless, due to the geometrical complexity of helically coiled tubes (curvature and presence of walls), the technique has not yet been applied to study such flows. Tomographic PIV (sometimes contracted to tomo-PIV) is based on the volume reconstruction of tracers seeded into the investigated flow. The light scattered from these tracers is recorded by two or more different camera viewing directions. Pairs of reconstructed volumes, calculated from these views and from two consecutive light pulses, are divided into interrogation boxes (subvolumes). The tracer pattern displacement is then computed by a $3 \mathrm{D}$ cross-correlation of pairs of interrogation 
boxes. Thus, the flow velocity is evaluated dividing the displacement by the known time delay between the light pulses.

Concerning numerical studies, so important for systematic investigation and performance optimization, an accurate prediction of the flow inside helically coiled tubes relies primarily on the limited existing "point" velocity measurements (Khot et al. 2019; Mansour et al. 2017; Mori and Nakayama 1967; Yamamoto et al. 2005). This is due to the lack of reference experimental data available in the laminar regime, where countless reactor applications operate, as well as transient and turbulent regimes, where many numerical simulations fail (Cioncolini and Santini 2006; Taylor 1929). Therefore, the present study concentrates on the experimental determination of 3C-3D velocity fields in a horizontally positioned helically coiled tube, using tomographic PIV technique at four flow conditions, comprehending fully laminar and transient regimes. Thus, validation data for numerical simulations will be available comporting full 3C-3D data sets in the complex geometry of a helically coiled tube. Additionally, in this study numerical simulations were performed in the same geometry and conditions as in the experiment to show the utility and applicability of such velocity and vortex structure data for further numerical parametric studies.

\section{Experimental set-up}

The experiments were carried out in a sinistrally coiled transparent glass helix of 15 coils with a $125-\mathrm{mm}$ long straight inlet. The inner tube diameter was $d=6 \mathrm{~mm}$, the coil diameter $D=27.4 \mathrm{~mm}$ and the pitch $b=13.33 \mathrm{~mm}$. The torsion-to-curvature ratio was $\lambda=0.15$. The cross section of the coiled tube was slightly elliptical because of the manufacturing process. The helix was placed in a decagonal prism made of polymethyl methacrylate (PMMA), with walls oriented orthogonal to the four camera viewing directions (Fig. 1). The decagonal prism was fully transparent, offering optical access from all sides.

Ammonium thiocyanate solution with 53.86\% mass-pervolume concentration, leading to a refractive index identical to that of the glass helix ( $n=1.468)$, was used as working fluid and to fill the decagonal prism. This refractive index matching technique strongly minimized optical distortions. The advantage of this solution is that its properties are near to those of water $\left(\rho=1132 \mathrm{~kg} / \mathrm{m}^{3}, \mu=1.349 \mathrm{mPa} \mathrm{s}\right)$. The flow was driven through the helix by gravity from a tank located $1.5 \mathrm{~m}$ above it, into a reservoir placed under the helix. The tank was filled with the working fluid before every measurement to avoid any pulsations induced by a pump. The maximum fluid-level variation in the tank during one experimental run was $26 \mathrm{~mm}$, leading to a pressure difference lower than $1.7 \%$ of the initial pressure. The flow rate was regulated with a screw hose clamp.

For the tomographic PIV measurements, four Imager sCMOS cameras (LaVision) with $2560 \times 2160$ pixels resolution equipped with Tokina $100 \mathrm{~mm}$ F2.8 MACRO lenses were linearly arranged. The lens apertures were set to $\mathrm{f} \# 32$, in order to obtain the entire width of the helix in focus. No Scheimpflug angles (lens tilt angles) were used, since the depth was equal to the height in the investigated volume. White Vestosint 2178 polyamide particles (mean diameter of $20 \mu \mathrm{m} ; \rho=1.23 \mathrm{~kg} / \mathrm{L}$ ) were added to the working fluid as tracers.

Homogenous illumination was delivered by 14 highpower COB LEDs (Luminus CXM-32) with a nominal power of $1960 \mathrm{~W}$, equally placed on the top, bottom, left and right side of the decagonal prism (Fig. 2). The pulsed a

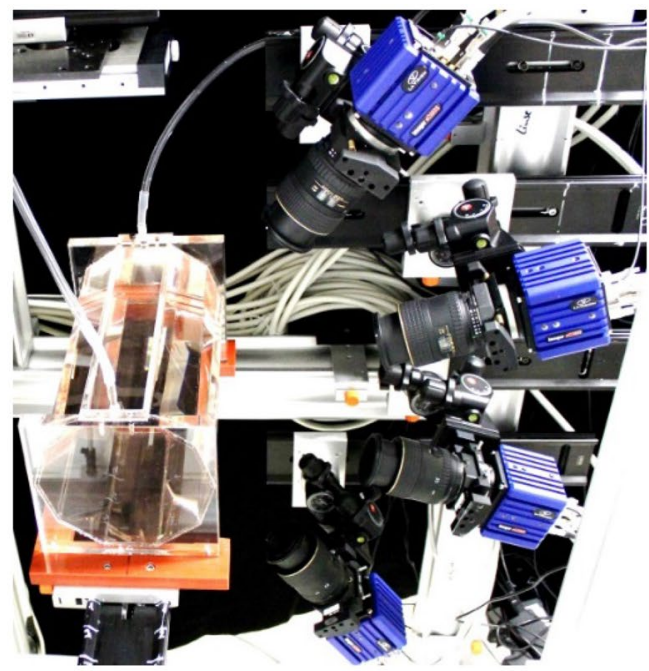

b

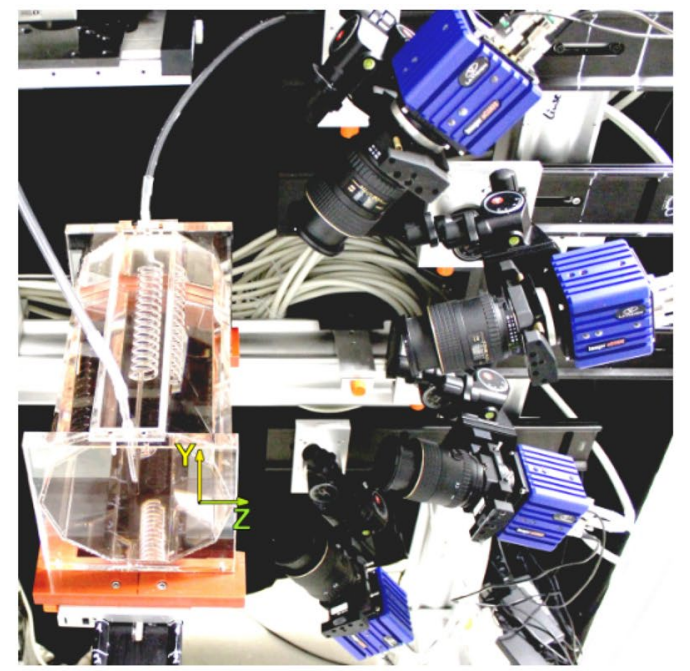

Fig. 1 Experimental set-up for tomographic PIV measurements. The helical coil is filled with the working fluid (a) and with air to be visible (b) 


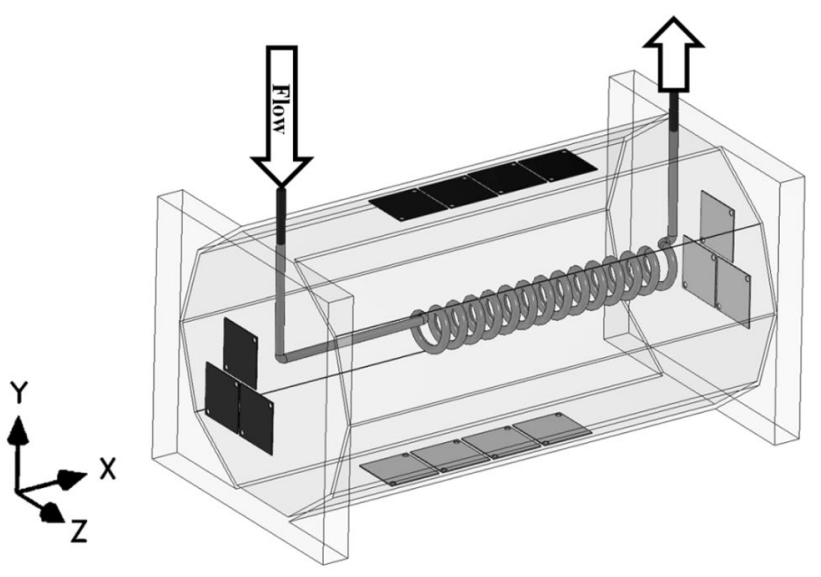

Fig. 2 LED (black and grey squares) arrangement around the decagonal prism

illumination duration of $50 \mu \mathrm{s}$ was short enough to avoid smearing of fast moving particles in the acquired images. The LEDs were operated slightly below their maximum recommended power values, in order to protect them from being overloaded, while providing high brightness and good signals of the particles on the camera sensors. The present LED system provided a simple, low-cost solution for high-power homogenous volume illumination. Nevertheless, contrarily to the monochromatic laser illumination, the used LEDs supplied white light, which is a combination of different wavelengths. This wide range of wavelengths led to small variation in the refractive index and to chromatic aberration effects (Martins et al. 2018), therefore slightly deteriorating the refractive index matching performance.

The experimental set-up and tomo-PIV processing were optimized according to Martins and co-workers (Martins et al. 2015) in order to obtain good quality measurements. The particle seeding concentration was kept low to compensate for the large particle diameter due to the large f-number used. The acquired images presented averaged seeding densities of 0.01 to 0.02 particles per pixel (ppp) with particle diameters of about 5 pixels, leading to a source density of approximately 0.3 . These values were below the recommended upper limits of $0.05 \mathrm{ppp}$ and source density of 0.35 found in the literature for accurately reconstructed volumes using a four-camera system and standard tomo-PIV processing (Discetti and Coletti 2018; Raffel et al. 2018).

Each camera recorded 1000 image pairs at a rate of $5 \mathrm{~Hz}$ for $\mathrm{Re}<1000$. At higher Reynolds number, the recording rate was increased to $10 \mathrm{~Hz}$. The time delay $\Delta \mathrm{t}$ between the two acquired frames and LED flashes was set between 300 and 12,000 $\mu$ s, also depending on the Reynolds number, in order to optimize the maximum particle displacement to about 8 pixels. The acquisition was synchronized by a programmable time unit driven by the DaVis 8.4 software
(LaVision), which was also employed to calibrate and to process the data.

The investigated helix region was divided into two fields of view (FOV), in order to increase the spatial resolution. The first FOV comprehended the straight inlet pipe and the first two coils, while the second consisted of the third to the fifth coil. During the measurements, the decagonal prism with the helical coil was traversed between the two views, while the cameras remained fixed.

\section{Volume calibration}

The crucial step for the quality of the tomographic reconstruction is the $3 \mathrm{D}$ volume calibration. It was performed in a two-step procedure: an initial calibration is followed by a correction based on the disparity field of triangulated particles. Initially, a 2D calibration target was placed into the filled decagonal prism without the helix. The dot pattern was recorded by the four cameras in 11 equally spaced parallel-depth positions in the investigated volume region, from $Z=-20 \mathrm{~mm}$ to $Z=20 \mathrm{~mm}$. A basic pinhole fit function was applied to map the physical coordinates into the image coordinates. This ground calibration was refined by a self-calibration process based on the particle disparity fields (Wieneke 2008) for all cases and both fields of views separately. First, the calibration target was removed and the fluid inside the prism, which contained few contaminants and particles, was stirred. A set of images was acquired of the region of interest. Subsequently, the glass helix was placed back to its position and a new set of images of flowing working fluid with tracer particles was recorded. For the self-calibration, the particle density in the recording images was reduced by filtering out the low intensity particles. A few iterations of self-calibration were performed with the combination of these two recorded sets, depending on the corresponding particle amount, intensity and velocity. In each self-calibration step, the calculated disparity maps were filtered and used to correct the actual calibration. At the end of the selfcalibration procedure, much less than 0.1-pixel root mean square triangulation error was achieved in all calibration planes, which is the recommended limit value to eventually yield a good quality reconstruction (Raffel et al. 2018).

\section{Tomo-PIV processing}

The recorded image sets were pre-processed in three main steps. The acquired raw images contained digital noise of the camera, fixed reflections due to the chromatic aberrations and scattered from particles stuck to the wall (Fig. 3a). Firstly, these artefacts were removed by a 3-image-wide symmetrical moving-average time filter over the image set 
Fig. 3 Image pre-processing steps: raw image (a), after moving average time filter (b), after 2D mask (c) and after particle intensity enhancement (d)
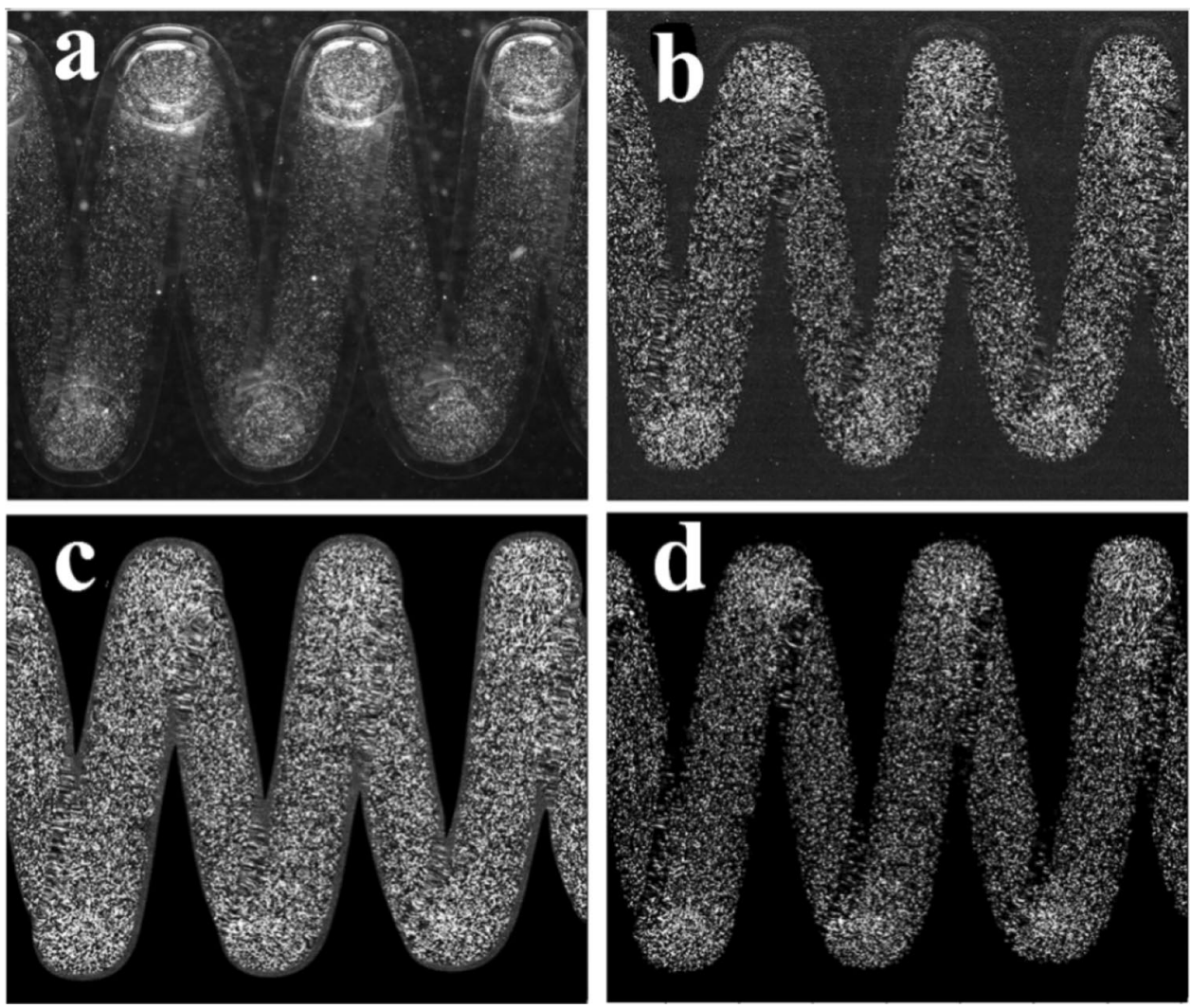

(Fig. 3b). Afterwards, a binarized algorithmic mask, based on Gaussian and sliding minimum filters, was applied on the images. In this way, the background intensity was set to ' 0 ', while the particle intensities were maintained (Fig. 3c). This pre-processing step significantly removed the noise and slightly reduced the particle image size. Both effects contribute to decrease the number of spurious particles ('ghost' particles) in the reconstructed volume (Martins et al. 2015). As a final step, the intensity and shape of the particles were enhanced by a Gaussian smoothing over $5 \times 5$ pixels, followed by a sharpening filter, and finally by zeroing the intensities below 8 intensity counts (Fig. 3d).

From the pre-processed images, pairs of 3D volumes were reconstructed from the four camera recordings. On the images with $2560 \times 2160$ pixels resolution, a $2 \times 2$ software binning was applied before the $3 \mathrm{D}$ reconstruction, because the available computational power (128 GB RAM) was not enough effective to reconstruct the 3D volume with the original spatial resolution. Accordingly, the resulting volume had $1380 \times 1238 \times 1364$ voxels and its volume reconstruction took around $40 \mathrm{~min}$ in a Xeon E5-1650v3 computer, while each of the $3 \mathrm{D}$ vector field calculations needed additionally 20 min.

The 3D volume reconstruction was first initialized with MinLOS, and then, five iterations of CSMART, followed by 16 iterations of SMART, were executed. More details about these algorithms can be found in the book of Raffel and co-workers (Raffel et al. 2018). In the intermediate reconstruction steps, a volume smoothing with strength of 0.5 and a volume threshold filter of 0.001 were enabled.

Unfortunately, the processing software allows only $2 \mathrm{D}$ masking on 3D volumes, which is not sufficient to mask such a complex geometry, as the present helical coil. Due to the manufacturing imperfectness of the glass helix, a 3D geometric masking with a CAD software was not possible either. For this reason, an in-house 3D algorithmic masking script was developed in MATLAB, based on thresholding a smoothed 3D particle distribution as following. First, the reconstructed volumes containing the instantaneous particle distribution were ensembled. Second, a cylinder, extruded along the $X$-axis and comprising the helical tube between its inner and outer diameter, was created (red circular lines in Fig. 4). It was based on projections of the ensemble of the reconstruction particle intensities onto the $Y Z-, X Y$ - and $X Z$-planes. Particles outside the cylinder thickness were removed. Third, volume intensities above a tiny threshold were set to ' 1 ', while the rest was set to ' 0 '. This reduced the number of ghost particles, which generally tend to have lower intensities (Raffel et al. 2018). Forth, the normalized volume was smoothed by a Gaussian filter with $20 \times 20 \times 20$ voxel size, in order to connect regions. Fifth, a low threshold was employed to create an initial mask of the approximate shape of the helix. Sixth, this initial mask was smoothed and then a final threshold, higher than the initial one, was applied 


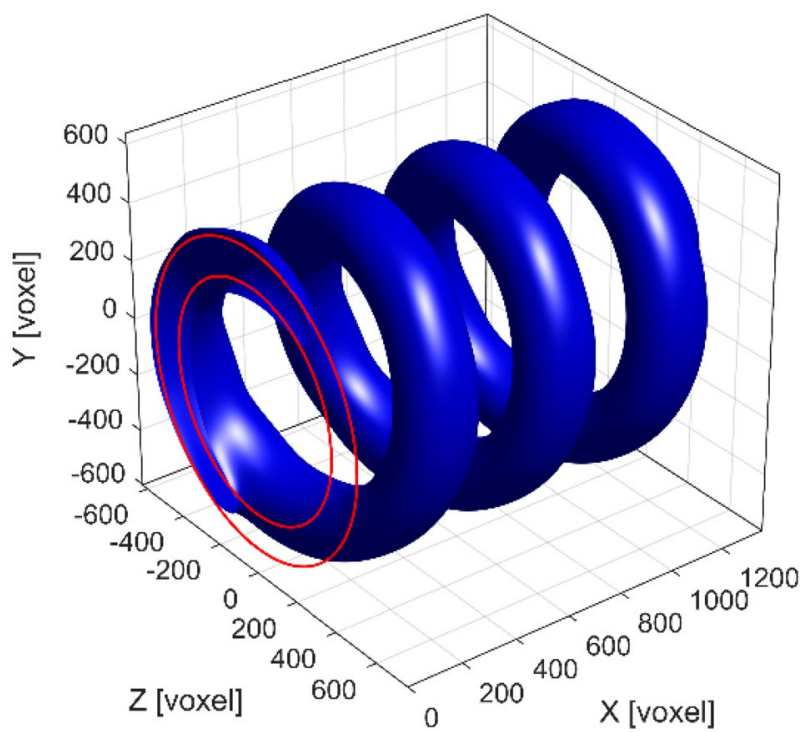

Fig. 4 Example of final 3D helical mask (blue isosurface of 0.8) with initial cylinder base (red circular lines) for the reconstructed volumes of the second field of view

to generate the 3D mask with similar dimensions of those of the actual helix (blue isosurface in Fig. 4). The present work adopted empirical initial and final thresholds of 0.05 and 0.8 for the 3D mask, respectively. The 3D helical mask was created for each field of view, and it was applied to the corresponding DaVis reconstructed volume files.

Geometric and algorithmic masks also allow for efficiently sparse storage, because only voxels inside the masks are related to the flow motion of interest. The sparse storage feature, although not implemented in DaVis 8.4 software, is available in many other software and programming libraries. In the present case, for example, the use of the described 2D algorithmic mask and 3D helical mask would reduce the storage requirements down to approximately $40 \%$ and $20 \%$ of the standard file size, respectively.

\section{Velocity evaluation}

For the velocity evaluation, pairs of $3 \mathrm{D}$ reconstructed volumes were subdivided into cubical interrogation boxes and a multi-pass direct volume cross-correlation between each corresponding interrogation box was applied only in regions where the box centre was inside the helical mask. This reduced significantly the computational processing time compared to that if the entire reconstructed volume is evaluated, because the helix occupies only circa $20 \%$ of the domain. The intensities outside the mask were set to ' 0 ' prior to the velocity computation. This seemed to improve the velocity field quality close to the walls, since the masking procedure reduces the amount of noise and the contribution of ghost particles outside the mask in the cross-correlation computations at these regions. Ghost particles are well known for biasing the velocity and deteriorating tomo-PIV measurements (Elsinga and Tokgoz 2014; Raffel et al. 2018). Preliminary tests at $\mathrm{Re}=220$ show root mean square differences between velocity evaluations with and without $3 \mathrm{D}$ mask around $0.02 \mathrm{~m} / \mathrm{s}$. Initial interrogation boxes of $48 \times 48 \times 48$-voxel size with $4 \times 4 \times 4$ binning (Discetti and Astarita 2012) were stepwise decreased towards final interrogation boxes of $32 \times 32 \times 32$-voxel size without binning and with $75 \%$ of overlap. The final 3D velocity field displayed a grid resolution of $8 \times 8 \times 8$ voxels (equivalent to $0.25 \mathrm{~mm}$ ). The present work employed two distinct approaches for computing the $3 \mathrm{C}-3 \mathrm{D}$ averaged velocity fields.

1. The first approach evaluated a time series of instantaneous velocity fields from pairs of instantaneous 3D reconstructed volumes, and then, time-averaged these velocity vector fields. This method is usually adopted in tomo-PIV (and also in standard PIV), and its resulted vector field will be termed as 'time-averaged velocity'.

2. The second approach was based on the ensemble crosscorrelation analysis. Velocity fields were computed from a pair of ensemble volumes averaged along the first exposure and second exposure of the reconstructed volume series. The approach is analogous from that of (Meinhart et al. 2000). This alternative method diminishes the computational time, because just one 3D velocity evaluation is necessary to obtain the $3 \mathrm{D}$ averaged velocity field. The resulted vector field using the second approach will be referred to as 'ensemble-volume velocity'.

Although in the present work, we used the same interrogation box size for the averaged velocity fields from instantaneous volume pairs and from ensemble-volume pairs, and the latter volumes allow for an evaluation of the fluid flow at considerably higher spatial resolution (smaller interrogation box) due to the higher amount of particles and better intensity contrast in the ensemble volume. This is a great benefit, because the seeding concentration in the working fluid for tomo-PIV measurements is normally lower and more restricted compared to that of planar or stereo PIV, due to the detrimental ghost particles generated as a 3D reconstruction artefact (Elsinga and Tokgoz 2014; Raffel et al. 2018). It is important to have in mind that this ensemble cross-correlation must only be applied when the flow is stationary, laminar or periodic. Although ensemble cross-correlation analysis has been efficiently employed for a long time in standard PIV with low particle image density recordings (Raffel et al. 2018), this approach has 
never been reported for applications in tomo-PIV measurements, as performed in the present work.

Besides the potential improvement in spatial resolution, the extension of the ensemble cross-correlation analysis to $3 \mathrm{D}$ measurements is of great interest, also regarding the computational cost required to perform standard interrogation calculations of 3D volumes. For example, using a direct cross-correlation without efficient approaches as fast Fourier transformation, sparse consideration or binning, the vector evaluation can be four to five orders of magnitude higher for tomo-PIV than that for planar PIV (Scarano 2013). The huge computational difference is a consequence of more elements in the interrogation box as well as more interrogation boxes and iterations for the $3 \mathrm{D}$ case.

\section{Numerical modelling}

In the present work, CFD simulations were performed in a similar sinistrally coiled helix with 15 coils (Fig. 5), using the commercial software Star-CCM + version 13. This code solves the transport equations of continuity and momentum balances based on the finite-volume method, which was used in several previous CFD simulations of our group (Jokiel et al. 2017; Khot et al. 2019; Mansour et al. 2017). The numerical domain has, in accordance with the experimental test-rig, a long inlet pipe before the helical tube. This was done to eliminate the numerical errors near the helical tube inlet and to confirm better flow prediction in the helix. Additionally, a Hagen-Poiseuille velocity profile was applied at the inlet surface, which again reduces the numerical errors associated with a uniform velocity profile near the domain inlet (Mansour et al. 2017). A constant pressure outlet boundary condition was always set at the helical tube outlet. For spacial discretization, hexahedral elements were generated with a total number of 15.4 million cells, with typical refinement near the wall to ensure accurate resolving of the boundary layer flow (Fig. 5).

Since preliminary studies proved that the vortices are stable (De Amicis et al. 2014; Kumar and Nigam 2005; Sheeba et al. 2019; Sreenivasan and Strykowski 1983), a steady-state set-up was used. All simulations were performed employing a laminar set-up, due to the investigated Reynolds number range $(20 \leq \mathrm{Re} \leq 1400)$. The segregated solver was applied to solve the flow with the SIMPLE algorithm for coupling velocity and pressure. As a discretization scheme for calculating the convection terms in the transport equations, the second-order upwind scheme was employed. In accordance with the horizontal orientation used in the experiments, the gravitational acceleration was active in all simulations. The fluid properties were set as those of the experiments. The solution was considered as converged when the normalized residuals of all conservation equations went below $10^{-6}$.

\section{Results}

In the present work, four different flow rates were investigated, from a fully laminar case at $\mathrm{Re}=20$ up to a transient case at $\operatorname{Re}=1400$ (Table 1 ). The estimated uncertainty in the flow rate was about 5-10\%. 3D instantaneous volumes were reconstructed for the two fields of view of each case. To this end, and because of the very high volume reconstruction and

Table 1 Flow parameters of the investigated cases

\begin{tabular}{lclrr}
\hline Case & $Q(\mathrm{~mL} / \mathrm{min})$ & $u(\mathrm{~m} / \mathrm{s})$ & $\mathrm{Re}$ & \multicolumn{1}{c}{$\mathrm{De}$} \\
\hline 1 & 7 & $3.97 \times 10^{-3}$ & 20 & 8 \\
2 & 47 & $2.78 \times 10^{-2}$ & 140 & 57 \\
3 & 74 & $4.37 \times 10^{-2}$ & 220 & 89 \\
4 & 472 & $2.78 \times 10^{-1}$ & 1400 & 567 \\
\hline
\end{tabular}

Fig. 5 Details of the numerical model and the mesh

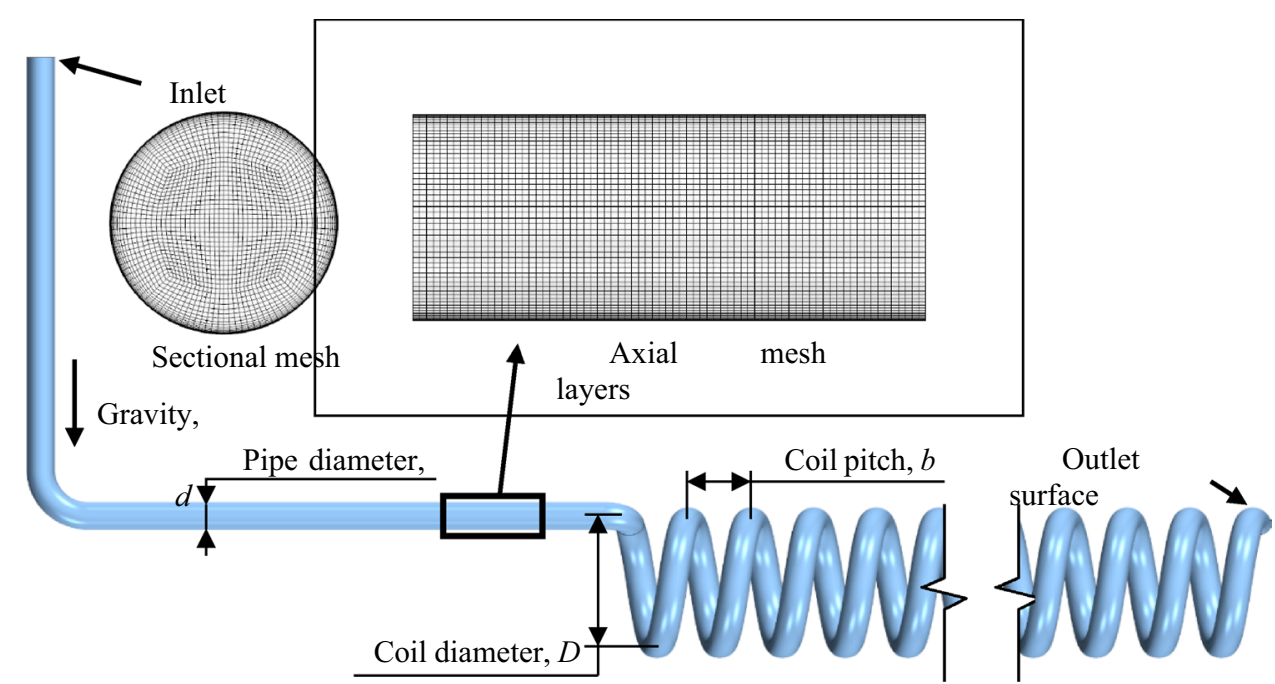


vector calculation time ( $1.5 \mathrm{~h}$ per field $)$, only every 10th image pair was processed, resulting in a set of 100 volume pairs. The mean velocity fields were calculated in two different ways: from the time average of 100 instantaneous velocity fields that were evaluated from the instantaneous volume pairs (time-averaged velocity), and from a single velocity field that was calculated from the ensemble pair of the same 100 instantaneous reconstructed volumes (ensemble-volume velocity).

The quality of the reconstructed volumes was assessed based on the light intensity signal-to-noise ratio (Martins et al. 2015; Raffel et al. 2018; Scarano 2013). This metric requires a top-hat illumination, a uniform seeding and a 'dark sample' (non-illuminated region or without seeding particles). First, an ensemble-volume containing the reconstructed intensity was computed time averaging the instantaneous reconstructed volumes. Then, the 'noise' from ghost particles and spurious reflections was estimated by averaging the intensities within the inner-diameter region of the helical mask, because no scattered light from actual particles was expected in this unseeded region (present dark sample). The 'signal', containing actual and ghost particles, was obtained by averaging the intensities within the helical mask. Finally, the signal-to-noise ratio was calculated, dividing these two average values. The computed signal-to-noise ratio was above 6 for all investigated cases, which is threefold higher than the suggested value for a good reconstruction quality (Raffel et al. 2018).

The reconstructed volume quality was additionally assessed by computing the normalized intensity variance (Novara and Scarano 2012). The volumes presented values above 25 , which are greater than the lower suggested level of 20 (Novara and Scarano 2012). In the present measurements, higher amount of ghost particles in the reconstructed volumes are expected in regions close to the tube wall and in regions where the light path refracted more times though the tube-liquid interface (rear side of the helical coil in Fig. 2), because of the slight chromatic effects leading to refractive index variation, as aforementioned (Sect. 2).

The velocity uncertainty was estimated based on the velocity divergence (Atkinson et al. 2011). The divergence was calculated from the velocity gradients along $X, Y$ and $Z$ directions, which were evaluated by a second-order central difference applied on the 3C-3D velocity field. The divergence of the velocity must be zero for an incompressible flow. Therefore, the root mean square of the divergence gives an estimate of the measurement errors. Lowest uncertainties were typically found in the centre of the tube, while highest uncertainties were near the tube wall, due to higher population of ghost particles and lower velocities. The average uncertainties in the first field of view were generally lower than in the second, possibly due to the calibration employed. The average standard uncertainty of the time-averaged velocity field of each studied case was below 0.17 voxel, while the uncertainty of the corresponding ensemble-volume velocity field was about twofold higher. These are good levels of uncertainty taking into account the complex geometry under study.

The standard velocity uncertainty based on the velocity divergence just described assumes uniform vector spacing along each direction and similar uncertainties in the velocity components, which sometimes is not realistic for tomo-PIV measurements. Wieneke (2015) proposed another uncertainty estimation approach based on the cross-correlation peaks used for the velocity calculation of each vector component. This standard velocity uncertainty approach was available in the DaVis software. The average of the uncertainty components computed from cross-correlation statistics perfectly agreed with the values computed using the velocity divergence. For instance, the spatially averaged uncertainties of the time-averaged velocity fields of the first $\mathrm{FOV}$ at $\mathrm{Re}=220$ were quantified based on the cross-correlation peaks as $0.0007,0.0013$ and $0.0017 \mathrm{~m} / \mathrm{s}$ for the $X, Y$ and $Z$ components, respectively, while the uncertainty based on the divergence was $0.0013 \mathrm{~m} / \mathrm{s}$. The lowest uncertainty was along $X$, aligned with the main flow direction, and the highest uncertainty was along $Z$, associated with some slight elongation of the reconstructed particles along the optical axis (Scarano 2013).

The 3D time-averaged velocity in the helical coil is shown in Fig. 6 for $R e=220$, visualized by velocity magnitude in horizontal ( $X Z$ plane) and vertical ( $X Y$ plane) slices. For that, the two fields of views were merged together at the second coil. The 3D helical mask is presented as a transparent grey isosurface. It is possible to observe that the inlet flow is axisymmetric, from the development along the long upstream straight pipe (Fig. 2). The flow enters in the coiled shape through an elbow and then the flow pattern turns into asymmetric, because of the evolving secondary flow.

The $Y$ and $Z$ velocity components close to the helix wall are presented in Fig. 7 for the same 3D velocity field of Fig. 6. This figure is useful to examine the consistency of the tomographic volume reconstruction and velocity measurements, which are obtained from the two, joined fields of view.

The flow evolution inside the helix can be seen in Fig. 8 through the extracted slices at different angles of the ensemble-volume velocity fields at $\mathrm{Re}=220$. In this figure, the rotation angle follows the helical sinistral revolution. The flow velocity in the straight inlet pipe is axisymmetric with a parabolic profile and agrees with that of a fully developed laminar pipe flow. Then, the flow enters into the coiled tube and due to the curvature the flow changes remarkably. The maximum flow velocity is located near the right bottom of the cross section at $45^{\circ}$, as an effect of the $90^{\circ}$ elbow downstream the straight inlet pipe. After one-eighth revolution 
Fig. 6 Time-averaged velocity inside the helically-coiled tube at $\mathrm{Re}=220$ and $\mathrm{De}=89$, visualized by vector-magnitude colour at horizontal and vertical slices
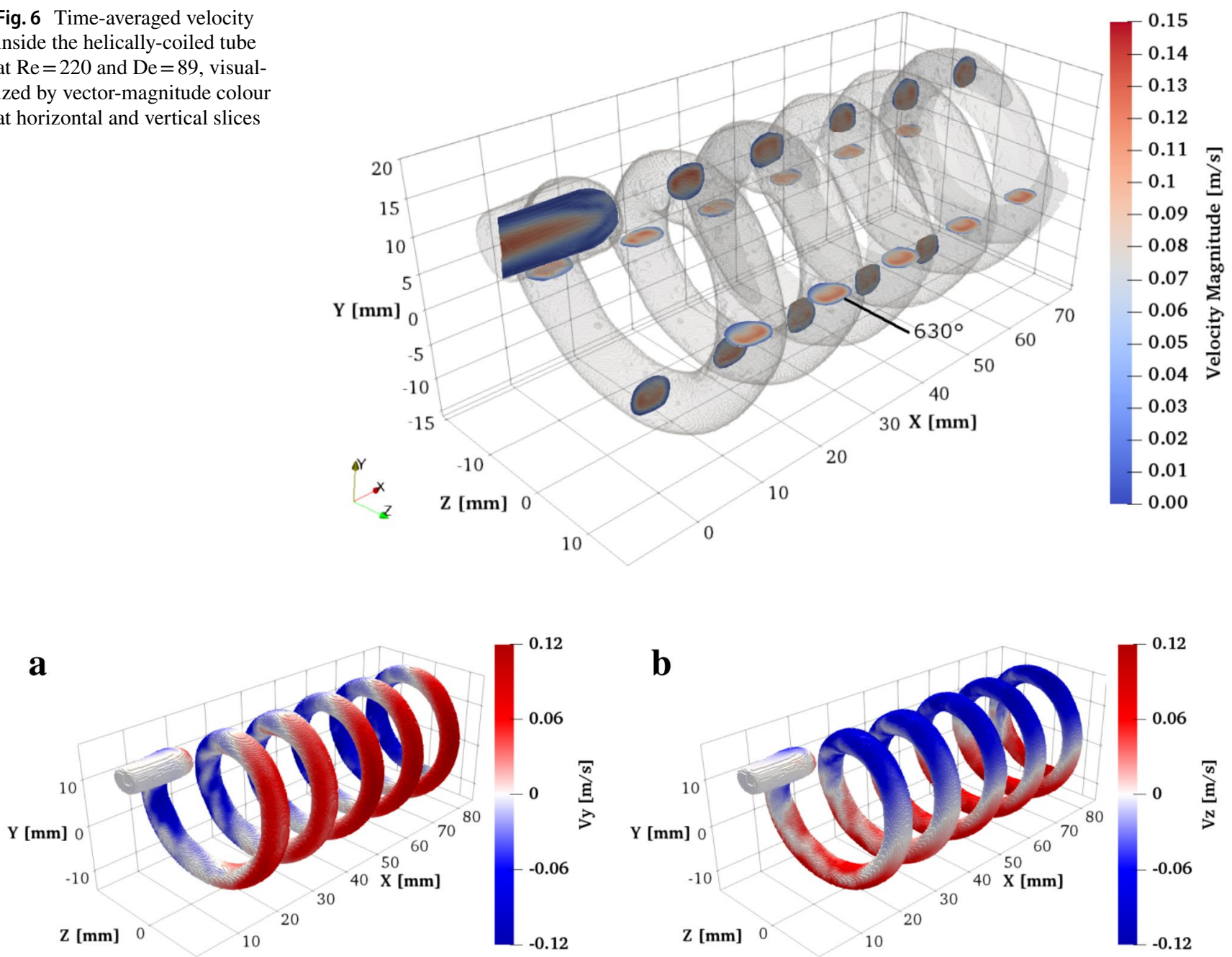

Fig. 7 Time-averaged velocity close to the wall of the helically-coiled tube at $\operatorname{Re}=220$ and $\mathrm{De}=89$ : $\mathbf{a} Y$-component and $\mathbf{b} Z$-component
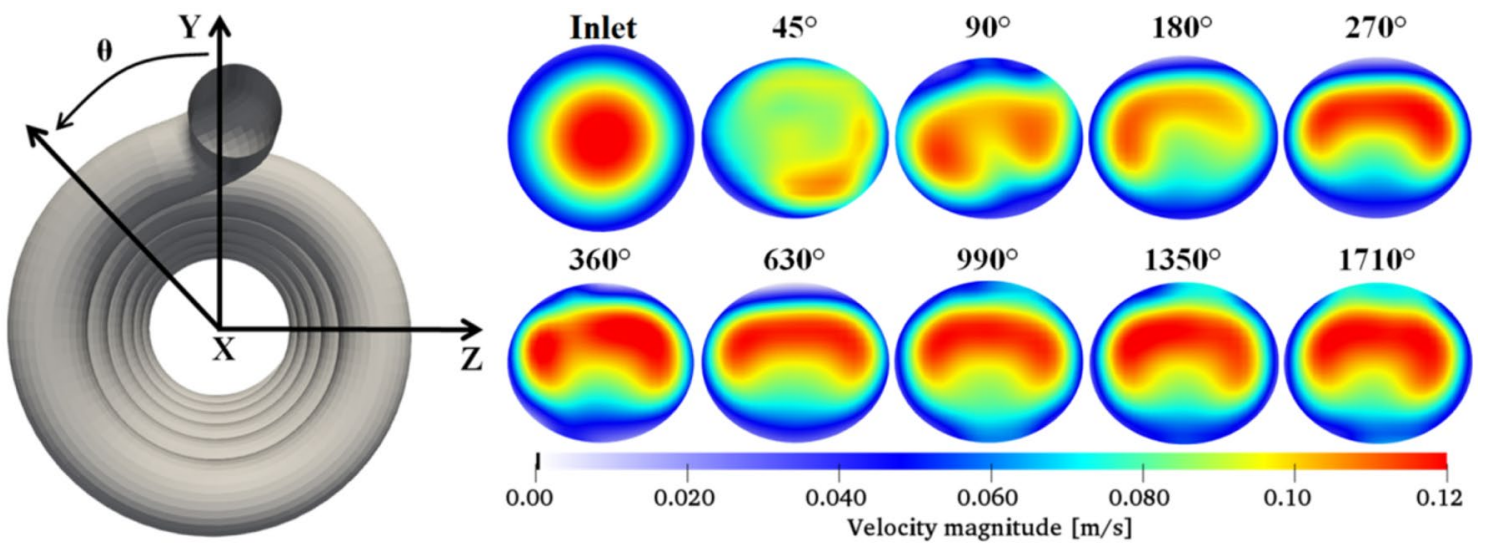

Fig. 8 Flow evolution in different cross sections of the ensemble-volume velocity along the helically coiled tube at $\operatorname{Re}=220$ and $\mathrm{De}=89$ 
downstream $\left(90^{\circ}\right)$, the flow becomes quasi-symmetric with respect to the vertical axis (helical radial direction), which clearly indicates the presence of secondary flows. Further downstream at around $270^{\circ}$, this secondary flow motions become persistent, stabilized by the helical coil geometry. After one full turn $\left(360^{\circ}\right)$, just slight changes can be observed in the flow structure, due to the stochastic nature of this flow and the uncertainty levels of the present measurements. The length required for fully established conditions and development of secondary flows is linked to the Reynolds number, the inlet geometry and the helix geometry. Except for $R e=20$, where no secondary flow is present, the same described flow development can be observed for all investigated laminar cases.

Since these tomo-PIV measurements provide the full velocity gradient tensor, 3D vortical structures can be detected, for example, by means of the 3D Q-criterion (Hunt et al. 1988), which is able to differentiate shear from rotational motions. The velocity spatial derivatives were computed based on the second-order central-difference scheme. Figure 9 presents a comparison between vortical structures at $\mathrm{Re}=220$ detected from the time-averaged velocity measurements (purple isosurfaces in the front of the helix) and those detected from the simulation (red isosurfaces in the rear of the helix) based on the 3D Q-criterion. The helical tube is presented as a transparent grey isosurface. A pair of strong counter-rotating vortices, the so-called Dean vortices, is easily identifiable by the detection algorithm. These vortices become stable downstream half-coil turn and span over the entire helix with persistent spatial separation between them. Excellent agreement is obtained between simulation and measurements regarding position and shape of the vortex pair. Minor discontinuities in the vortices detected from the measurement data reflect the increase in the uncertainty

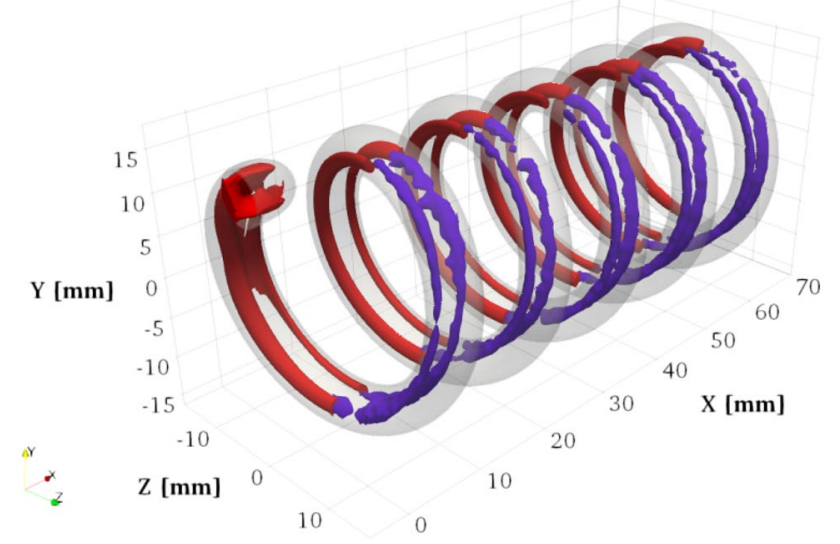

Fig. 9 Dean vortices detected by 3D Q-criterion inside the helicallycoiled tube at $\mathrm{Re}=220$ and $\mathrm{De}=89$ as obtained from the time-averaged measurements (purple isosurfaces) and from the simulation (red isosurfaces) and noise due to the derivatives, since no filtering was applied.

Time-average velocity measurements of all investigated cases (from $\operatorname{Re}=20$ to $\operatorname{Re}=1400$ ) at the position $630^{\circ}$ (Fig. 6) are compared with computational simulations in Fig. 10. The figure shows colour plots of normalized velocity magnitude $v / v_{\max }$ and some vortex detection criteria as $2 \mathrm{D}$ vorticity (out-of-plane vorticity), 3D Q-criterion and 2D line integral convolution (surface LIC) (Cabral and Leedom 1993), produced using ParaView software. A similar representation can be found in the supplementary material also for the comparison of the ensemble-volume velocity measurements. In the first investigated case at $\operatorname{Re}=20$ and $\mathrm{De}=8$ (first line in Fig. 10), the flow shows fully laminar nature, with the maximum flow velocity coinciding with the tube centre. Deviations from the axisymmetry are attributed to the uncertainty level and the ghost particles close to the tube wall. No secondary flow pattern is observed at this low Reynolds number, in agreement with the simulations. At $\operatorname{Re}=140$ and $\mathrm{De}=57$, a dual peak velocity distribution appears, with its maximum displaced towards the outer helix wall, forming a 'bean' shape. Two counter-rotating vortices also appear as a result of this secondary flow motions. With increasing flow velocity, these secondary vortices become more stable and stronger. The coiled section stabilized the flow inhibiting instabilities (Sreenivasan and Strykowski 1983); therefore, the formed rotation motions are stable in time and space. All employed vortex detection criteria seem capable to identify the presence of such vortices in the visualized cross section. At $\mathrm{Re}=220$ and $\mathrm{De}=89$, the maximum velocity regions shift more towards the outer wall, while the two counter-rotating vortices move towards the inner helix wall and slightly outwards to the side walls. This motion continuous with increasing Reynolds number, and, at $\operatorname{Re}=1400$ and $\mathrm{De}=567$, the cores of the vortex pair are pushed more close to the wall, which causes elongation of the cross section of these vortical structures. Besides the change in the vortices, the flow pattern changes as well. Almost half of the tube cross section is occupied by the high-velocity regions, which are extended downwards along the walls.

The colour plots from the measured velocity fields in Fig. 10 show an excellent agreement with those from the numerical simulations, except at $\mathrm{Re}=1400$ and $\mathrm{De}=567$, where the currently employed RANS simulation seems incapable to resolve the more intricate flow pattern that arises in the transient regime, slightly overestimating the velocity magnitude. The reason for this discrepancy may be found in the changing of secondary flow structures, which seems not fully stable anymore. Other PIV and laser-induced florescence experiments performed by our group (not presented here) using different helical geometries showed that, above $\operatorname{Re}=600$, even additional vortical structures, known as Lyne 


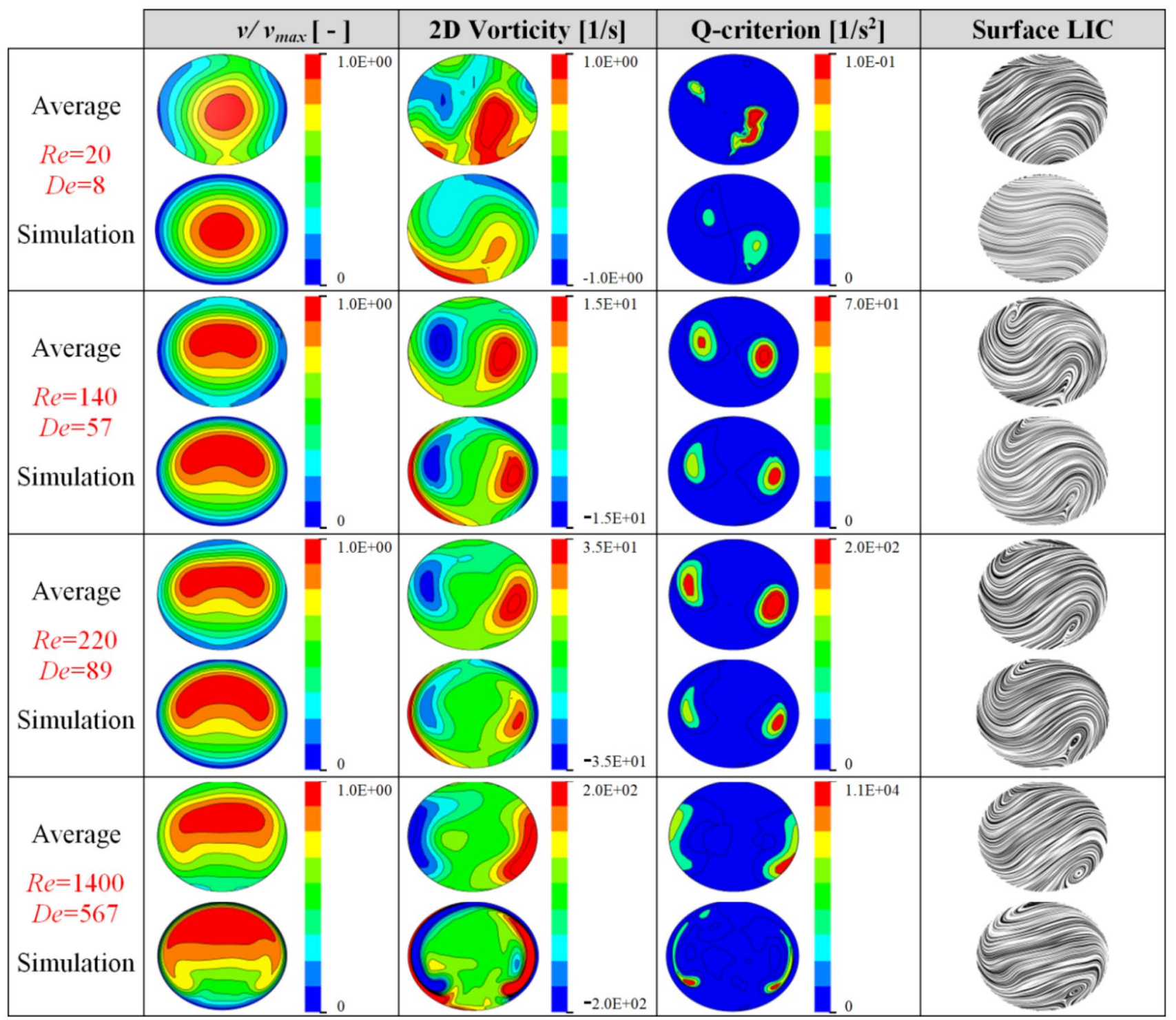

Fig. 10 Colour plots of normalized velocity magnitude, out-of-plane vorticity, 3D Q-criterion and 2D line integral convolution (surface LIC) at $630^{\circ}$ for time-averaged measurements and simulations

vortices in the literature (Dennis and Ng 1982; Lyne 1971; Yanase et al. 1989), can appear close to the outer wall. These vortical structures are not always stable and seem to have a stochastic nature, which is troublesome to be predicted with RANS numerical simulations. The transitional regime with growing instabilities can extend to much higher Reynolds number than that of a straight pipe due to the curvature of the tube, delaying the transition to a fully turbulent flow (Cioncolini and Santini 2006; Sreenivasan and Strykowski 1983; Taylor 1929).

For a quantitative comparison of time-averaged and ensemble-volume velocity fields with the corresponding numerical simulations, profiles of the three velocity components were extracted along the vertical and horizontal lines of the cross sections $\left(630^{\circ}\right)$, with lines crossing at the tube centre (Fig. 11). These lines correspond to the $X$ and $Z$ directions of the helix (Fig. 6). The profiles are normalized by the velocity magnitude.

The flow governing velocity component is $v_{y}$, which is the out-of-plane component of the $X Z$ slice (position $630^{\circ}$ in Fig. 6). The horizontal and vertical velocity profiles at $\mathrm{Re}=20$ agree with a velocity profile of a developed laminar flow, with symmetric profiles of all velocity components. It is worth mentioning that $v_{x}$ and $v_{y}$ are not zero, because the orientation of the $X Z$ plane is not orthogonal to the helical tube. The experimental velocity profiles show, in the low Reynolds number cases, inaccuracies close to the wall, since the locally low fluid displacement accumulated tracer 


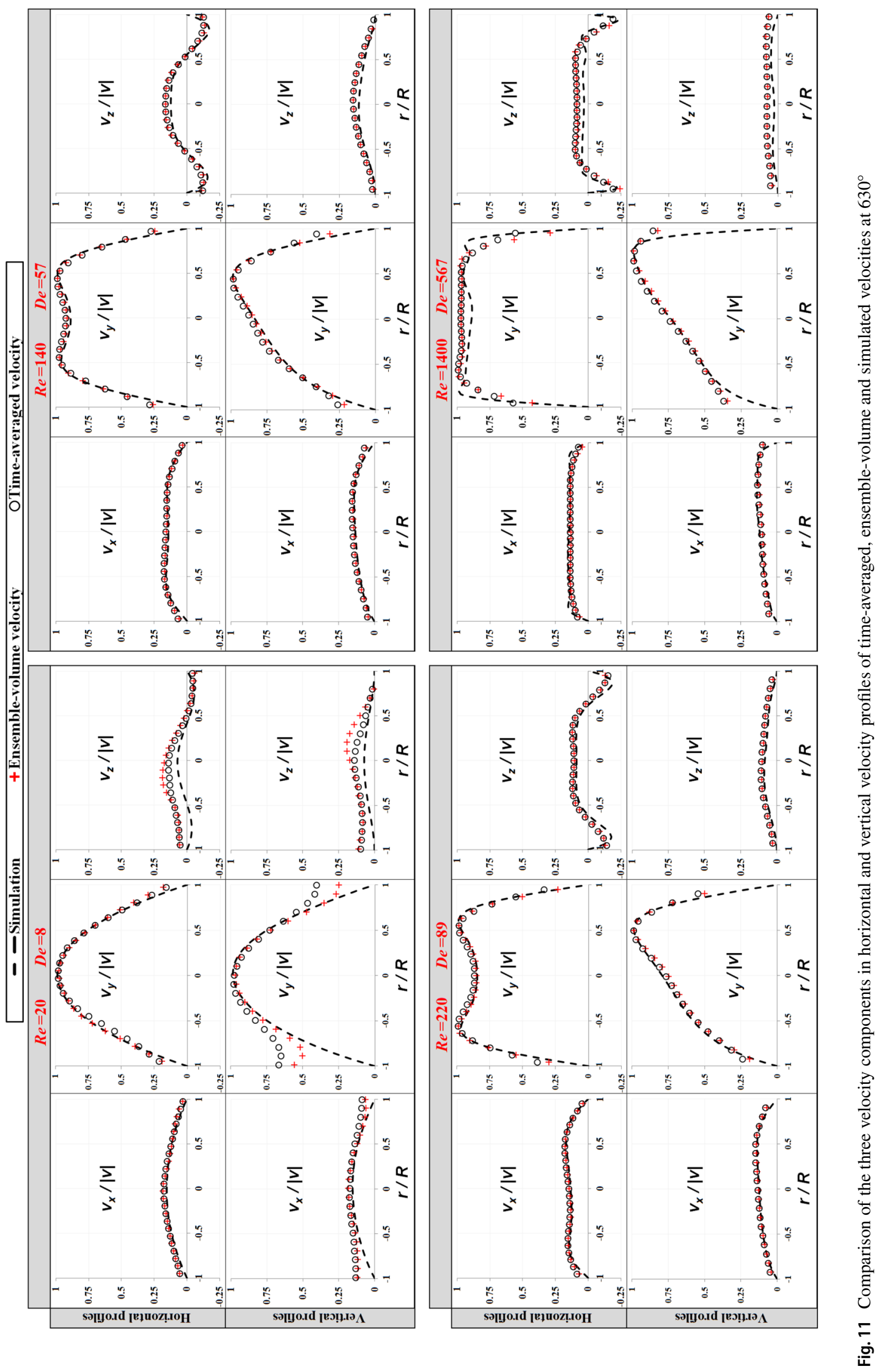


particles onto the wall surface. The highest relative velocity uncertainties are observed for the $v_{z}$ component, as explained previously. With increasing Reynolds number, the horizontal velocity profiles remain symmetric, but, according to the evolving secondary flow, the single peak of the $v_{y}$ component splits into two peaks that move away from each other towards the tube wall. Due to the developing Dean vortices, the $v_{z}$ velocities in the horizontal profile increase along the positive direction in the centre of the cross section and a back-flow develops near the side tube walls. Regarding the vertical profiles, the maxima of the $v_{y}$ component shifts towards the outer wall of the helix at the same time as the $v_{x}$ maxima move towards the right wall (in the positive direction of the helix axis). At $\mathrm{Re}=140$, the two counter rotating vortices occupy the tube cross section, as it is clearly observable in the horizontal $v_{z}$ profiles, as well as in the $2 \mathrm{D}$ vorticity images (Fig. 10). With increasing flow rate, these vortices are pushed more to the side walls and provoke a much steeper velocity gradient. This gradient may not be completely resolved by the measurements, also due the aforementioned sticking of particles to the wall. In the first three investigated cases, the experiments and simulations show a good agreement, but at the highest Reynolds number the differences are noticeable. The standard steady-state RANS simulation seems not capable to fully predict this transitional flow even in this early stage, as already discussed in Fig. 10.

\section{Conclusions}

This is the first systematic study concerning 3C-3D velocity measurements by tomographic PIV in a helical coil, to the authors' best knowledge. A wide range of Reynolds numbers from 20 to 1400 and Dean numbers from 8 to 567 was investigated and the secondary Dean vortices could be observed.

The applied measurement technique has required a strong and homogenous illumination, which was supported by high-power LEDs. The LED arrays provided sufficient light for the measurements and therefore are a good alternative to the costly laser volume illumination. The refractive index matching of the surrounding and working fluid to the helix material allowed for the optical measurements inside the complex helix geometry.

Regarding the reconstructed volume masking, a non-traditional 3D masking procedure of the complex geometry, using the reconstructed particle intensities, was proposed. This essential processing step, prior to the velocity evaluation, in order to reduce the amount of noise and ghost particles, enhances the final quality of the vector fields and additionally reduces the computational processing time and storage space of sparse files.
For the evaluation of the velocity fields, two approaches were applied: time-averaged velocity calculation from instantaneous velocity fields and velocity field determination by cross-correlation from a pair of the ensemble of instantaneous reconstructed volumes. Good reconstructed volume quality and velocity field accuracy were obtained in both cases and both approaches lead to similar velocity fields for laminar and Dean regimes, when the flow can be considered stationary. The ensemble-volume approach offers advantages compared to the traditional time-averaged calculation: it is computationally faster (particularly in 3D applications) and can lead to better spatial resolution. Therefore, ensemblevolume calculation should be employed more often in tomographic PIV evaluations of stationary flows.

The present flow field investigation inside the horizontally positioned helical tube shows fully flow establishment after $360^{\circ}$ and secondary motions with the presence of a pair of counter-rotating vortices characterizing the Dean regime from $\operatorname{Re}=140$ and $\mathrm{De}=57$ to $\operatorname{Re}=1400$ and $\mathrm{De}=567$. The present vortices are stable in time and space from $\mathrm{Re}=140$ and $\mathrm{De}=57$ to $\mathrm{Re}=220$ and $\mathrm{De}=89$. The experimental results are in excellent agreement with numerical simulations of laminar flows also performed in the scope of this work. The standard steady-state RANS simulation seems not fully able to predict the studied transitional flow $(\operatorname{Re}=1400$ and $\mathrm{De}=567$ ), due to the more intricate secondary flow. The present flow characterization and tomo-PIV measurement data can be further used to validate numerical models and other computational simulations, in particular for a systematic characterization of the influence of geometrical parameters and the role of Dean vortices on the heat and mass-transfer performance of helically coiled reactors.

Acknowledgements Open Access funding provided by Projekt DEAL. The financial support of the Deutsche Forschungsgemeinschaft (DFG, German Research Foundation) - TRR 63 "Integrated Chemical Processes in Liquid Multiphase Systems" (subproject B1) - 56091768 is gratefully acknowledged (Gefördert durch die Deutsche Forschungsgemeinschaft (DFG) - TRR 63 "Integrierte chemische Prozesse in flüssigen Mehrphasensystemen" (Teilprojekt B1) - 56091768). Authors would also like to thank their students Moritz Meist, Damaris Pohl and Dávid Faragó for their participation in the experiments.

Open Access This article is licensed under a Creative Commons Attribution 4.0 International License, which permits use, sharing, adaptation, distribution and reproduction in any medium or format, as long as you give appropriate credit to the original author(s) and the source, provide a link to the Creative Commons licence, and indicate if changes were made. The images or other third party material in this article are included in the article's Creative Commons licence, unless indicated otherwise in a credit line to the material. If material is not included in the article's Creative Commons licence and your intended use is not permitted by statutory regulation or exceeds the permitted use, you will need to obtain permission directly from the copyright holder. To view a copy of this licence, visit http://creativecommons.org/licenses/by/4.0/. 


\section{References}

Acharya N, Sen M, Hsueh-Chia C (1992) Heat transfer enhancement in coiled tubes by chaotic mixing. Int J Heat Mass Transf 35:24752489. https://doi.org/10.1016/0017-9310(92)90090-f

Adler M (1934) Strömung in gekrümmten Rohren. ZAMM Zeitschrift für Angewandte Mathematik und Mechanik 14:257-275. https:// doi.org/10.1002/zamm.19340140502

Agrawal Y, Talbot L, Gong K (1978) Laser anemometer study of flow development in curved circular pipes. J Fluid Mech 85:497-518. https://doi.org/10.1017/s0022112078000762

Atkinson C, Coudert S, Foucaut JM, Stanislas M, Soria J (2011) The accuracy of tomographic particle image velocimetry for measurements of a turbulent boundary layer. Exp Fluids 50:1031-1056. https://doi.org/10.1007/s00348-010-1004-z

Brito M, Sanches P, Ferreira RML, Covas DIC (2016) Experimental study of the transient flow in a coiled pipe using PIV. J Hydraul Eng 143:04016087. https://doi.org/10.1061/(asce)hy.19437900.0001253

Bulusu KV, Plesniak MW (2018) Insights on arterial secondary flow structures and vortex dynamics gained using the MRV technique. Int J Heat Fluid Flow 73:143-153. https://doi.org/10.1016/j.ijhea tfluidflow.2018.08.002

Cabral B, Leedom LC (1993) Imaging vector fields using line integral convolution. In: Proceedings of the 20th annual conference on computer graphics and interactive techniques, pp 263-270. https ://doi.org/10.1145/166117.166151

Cioncolini A, Santini L (2006) An experimental investigation regarding the laminar to turbulent flow transition in helically coiled pipes. Exp Therm Fluid Sci 30:367-380. https://doi.org/10.1016/j.expth ermflusci.2005.08.005

De Amicis J, Cammi A, Colombo LPM, Colombo M, Ricotti ME (2014) Experimental and numerical study of the laminar flow in helically coiled pipes. Prog Nucl Energy 76:206-215. https://doi. org/10.1016/j.pnucene.2014.05.019

Dean WR (1927) XVI.Note on the motion of fluid in a curved pipe. Lond Edinb Dublin Philos Mag J Sci 4:208-223. https://doi. org/10.1080/14786440708564324

Dean WR (1928) LXXII.The stream-line motion of fluid in a curved pipe (Second paper). Lond Edinb Dublin Philos Mag J Sci 5:673695. https://doi.org/10.1080/14786440408564513

Dennis SCR, Ng M (1982) Dual solutions for steady laminar flow through a curved tube. Q J Mech Appl Math 35:305-324. https:// doi.org/10.1093/qjmam/35.3.305

Discetti S, Astarita T (2012) Fast 3D PIV with direct sparse crosscorrelations. Exp Fluids 53:1437-1451. https://doi.org/10.1007/ s00348-012-1370-9

Discetti S, Coletti F (2018) Volumetric velocimetry for fluid flows. Meas Sci Technol 29:ARTN 042001. https://doi. org/10.1088/1361-6501/aaa571

Elsinga GE, Tokgoz S (2014) Ghost hunting —an assessment of ghost particle detection and removal methods for tomographic-PIV. Meas Sci Technol 25:084004. https://doi.org/10.1088/09570233/25/8/084004

Elsinga GE, Scarano F, Wieneke B, van Oudheusden BW (2006) Tomographic particle image velocimetry. Exp Fluids 41:933-947. https://doi.org/10.1007/s00348-006-0212-z

Enayet MM, Gibson MM, Taylor AMKP, Yianneskis M (1982) Laser-Doppler measurements of laminar and turbulent flow in a pipe bend. Int J Heat Fluid Flow 3:213-219. https://doi. org/10.1016/0142-727x(82)90024-8

Eustice J (1997) Experiments on stream-line motion in curved pipes. Proc R Soc Lond Ser A Contain Pap Math Phys Character 85:119 131. https://doi.org/10.1098/rspa.1911.0026
Fukushima E (1999) Nuclear magnetic resonance as a tool to study flow. Annu Rev Fluid Mech 31:95-123. https://doi.org/10.1146/ annurev.fluid.31.1.95

Hunt JCR, Wray AA, Moin P (1988) Eddies, streams and convergence zones in turbulent flows. In: Proceedings of the summer program center turbulence research, NASA Ames. pp 193-207

Jokiel M, Wagner LM, Mansour M, Kaiser NM, Zahringer K, Janiga G, Nigam KDP, Thevenin D, Sundmacher K (2017) Measurement and simulation of mass transfer and backmixing behavior in a gas-liquid helically coiled tubular reactor. Chem Eng Sci 170:410-421. https://doi.org/10.1016/j.ces.2017.01.027

Kalpakli A, Örlü R (2013) Turbulent pipe flow downstream a $90^{\circ}$ pipe bend with and without superimposed swirl. Int J Heat Fluid Flow 41:103-111. https://doi.org/10.1016/j.ijheatfluidflow.2013.01.003

Kalpakli Vester A, Sattarzadeh SS, Örlü R (2016) Combined hot-wire and PIV measurements of a swirling turbulent flow at the exit of a $90^{\circ}$ pipe bend. $\mathrm{J}$ Vis 19:261-273. https://doi.org/10.1007/s1265 0-015-0310-1

Khot P, Mansour M, Thévenin D, Nigam KDP, Zähringer K (2019) Improving the mixing characteristics of coiled configurations by early flow inversion. Chem Eng Res Des 146:324-335. https://doi. org/10.1016/j.cherd.2019.04.016

Koutsky JA, Adler RJ (1964) Minimisation of axial dispersion by use of secondary flow in helical tubes. Can J Chem Eng 42:239-246. https://doi.org/10.1002/cjce.5450420602

Kováts P, Pohl D, Thévenin D, Zähringer K (2018) Optical determination of oxygen mass transfer in a helically-coiled pipe compared to a straight horizontal tube. Chem Eng Sci 190:273-285. https:// doi.org/10.1016/j.ces.2018.06.029

Ku DN (1997) Blood flow in arteries. Annu Rev Fluid Mech 29:399_ 434. https://doi.org/10.1146/annurev.fluid.29.1.399

Kumar V, Nigam KDP (2005) Numerical simulation of steady flow fields in coiled flow inverter. Int J Heat Mass Transf 48:48114828. https://doi.org/10.1016/j.ijheatmasstransfer.2005.05.037

Kurt SK, Gelhausen MG, Kockmann N (2015) Axial dispersion and heat transfer in a milli/microstructured coiled flow inverter for narrow residence time distribution at laminar flow. Chem Eng Technol 38:1122-1130. https://doi.org/10.1002/ceat.201400515

Liang D, Zhang SF (2014) A contraction-expansion helical mixer in the laminar regime. Chin J Chem Eng 22:261-266. https://doi. org/10.1016/S1004-9541(14)60035-5

Liou TM (1992) Flow visualization and LDV measurement of fully developed laminar flow in helically coiled tubes. Exp Fluids 13:332-338. https://doi.org/10.1007/bf00209507

Lyne WH (1971) Unsteady viscous flow in a curved pipe. J Fluid Mech 45:13-31. https://doi.org/10.1017/s0022112071002970

Mansour M, Liu Z, Janiga G, Nigam KDP, Sundmacher K, Thévenin D, Zähringer K (2017) Numerical study of liquid-liquid mixing in helical pipes. Chem Eng Sci 172:250-261. https://doi. org/10.1016/j.ces.2017.06.015

Martins FJWA, Foucaut JM, Thomas L, Azevedo LFA, Stanislas M (2015) Volume reconstruction optimization for tomo-PIV algorithms applied to experimental data. Meas Sci Technol 26:Artn 085202. https://doi.org/10.1088/0957-0233/26/8/085202

Martins FJWA, da Silva CC, Lessig C, Zähringer K (2018) Ray-tracing based image correction of optical distortion for PIV measurements in packed beds. J Adv Opt Photon 1:71-94. https://doi. org/10.3970/jaop.2018.03870

Meinhart CD, Wereley ST, Santiago JG (2000) A PIV algorithm for estimating time-averaged velocity fields. J Fluids Eng 122:285-289

Mejia Mendez DL, Lemaitre C, Castel C, Ferrari M, Simonaire H, Favre E (2017) Membrane contactors for process intensification of gas absorption into physical solvents: impact of dean 
vortices. J Membr Sci 530:20-32. https://doi.org/10.1016/j.memsc i.2017.02.016

Mishra P, Gupta S (1979) Momentum transfer in curved pipes. 1. Newtonian fluids. Ind Eng Chem Process Des Dev 18:130-137

Mori Y, Nakayama W (1967) Study of forced convective heat transfer in curved pipes (2nd report, turbulent region). Int J Heat Mass Transf 10:37-59. https://doi.org/10.1016/0017-9310(67)90182-2

Moulin P, Rouch JC, Serra C, Clifton MJ, Aptel P (1996) Mass transfer improvement by secondary flows: Dean vortices in coiled tubular membranes. J Membr Sci 114:235-244. https://doi. org/10.1016/0376-7388(95)00323-1

Novara M, Scarano F (2012) Performances of motion tracking enhanced Tomo-PIV on turbulent shear flows. Exp Fluids 52:1027-1041. https://doi.org/10.1007/s00348-011-1187-y

Raffel M, Willert CE, Scarano F, Kähler CJ, Wereley ST, Kompenhans J (2018) Particle image velocimetry, 3rd edn. Springer, Berlin. ISBN 978-3-319-68851-0

Sakakibara J, Machida N (2012) Measurement of turbulent flow upstream and downstream of a circular pipe bend. Phys Fluids 24:Artn 041702. https://doi.org/10.1063/1.4704196

Saxena AK, Nigam KDP (1983) Laminar dispersion in helically coiled tubes of square cross-section. Can J Chem Eng 61:53-57. https:// doi.org/10.1002/cjce.5450610109

Scarano F (2013) Tomographic PIV: principles and practice. Meas Sci Technol 24:Artn 012001. https://doi.org/10.1088/09570233/24/1/012001

Sheeba A, Abhijith CM, Prakash MJ (2019) Experimental and numerical investigations on the heat transfer and flow characteristics of a helical coil heat exchanger. Int J Refrig Revue Internationale Du Froid 99:490-497. https://doi.org/10.1016/j.ijrefrig.2018.12.002

Soni B, Lindley C, Thompson D (2009) The combined effects of nonplanarity and asymmetry on primary and secondary flows in the small bronchial tubes. Int J Numer Methods Fluids 59:117-146. https://doi.org/10.1002/fld.1802

Sreenivasan KR, Strykowski PJ (1983) Stabilization effects in flow through helically coiled pipes. Exp Fluids 1:31-36. https://doi. org/10.1007/bf00282264

Sudo K, Sumida M, Hibara H (1998) Experimental investigation on turbulent flow in a circular-sectioned 90-degree bend. Exp Fluids 25:42-49. https://doi.org/10.1007/s003480050206
Sudo K, Sumida M, Hibara H (2000) Experimental investigation on turbulent flow through a circular-sectioned $180^{\circ}$ bend. Exp Fluids 28:51-57. https://doi.org/10.1007/s003480050007

Taylor GI (1929) The criterion for turbulence in curved pipes. Proc R Soc Lond Ser A Contain Pap Math Phys Character 124:243-249. https://doi.org/10.1098/rspa.1929.0111

Taylor GI (1997) The criterion for turbulence in curved pipes. Proc R Soc Lond Ser A Contain Pap Math Phys Character 124:243-249. https://doi.org/10.1098/rspa.1929.0111

Tohidi A, Ghaffari H, Nasibi H, Mujumdar AS (2015) Heat transfer enhancement by combination of chaotic advection and nanofluids flow in helically coiled tube. Appl Therm Eng 86:91-105. https:// doi.org/10.1016/j.applthermaleng.2015.04.043

Vashisth S, Kumar V, Nigam KDP (2008) A review on the potential applications of curved geometries in process industry. Ind Eng Chem Res 47:3291-3337. https://doi.org/10.1021/ie701760h

Wieneke B (2008) Volume self-calibration for 3D particle image velocimetry. Exp Fluids 45:549-556

Wieneke B (2015) PIV uncertainty quantification from correlation statistics. Meas Sci Technol 26:Artn 074002. https://doi. org/10.1088/0957-0233/26/7/074002

Yamamoto K, Hayamizu Y, Matsuoka Y, Ohta K (2005) Measurement of flow velocity and turbulence in a helically curved pipe. Trans Jpn Soc Mech Eng Ser B 71:1278-1285. https://doi.org/10.1299/ kikaib.71.1278

Yanase S, Goto N, Yamamoto K (1989) Dual solutions of the flow through a curved tube. Fluid Dyn Res 5:191-201. https://doi. org/10.1016/0169-5983(89)90021-x

Yasuo M, Wataru N (1965) Study on forced convective heat transfer in curved pipes. Int J Heat Mass Transf 8:67-82. https://doi. org/10.1016/0017-9310(65)90098-0

Publisher's Note Springer Nature remains neutral with regard to jurisdictional claims in published maps and institutional affiliations.

\section{Affiliations}

\section{Péter Kováts ${ }^{1} \cdot$ Fabio J. W. A. Martins ${ }^{1,2} \cdot$ Michael Mansour $^{1,3} \cdot$ Dominique Thévenin $^{1} \cdot$ Katharina Zähringer $^{1}$ (D)}

1 Laboratory of Fluid Dynamics and Technical Flows, Institute of Fluid Dynamics and Thermodynamics (ISUT), Otto-vonGuericke-University, Magdeburg, Magdeburg, Germany

2 Laboratory of Thermodynamics and Combustion, Institute of Fluid Dynamics and Thermodynamics (ISUT), Otto-von-Guericke-University, Magdeburg, Magdeburg, Germany
3 Mechanical Power Engineering Department, Faculty of Engineering - Mattaria, Helwan University, Cairo, Egypt 